\title{
La « Route de l'esclave » de Ouidah : espace de négociation des mémoires collectives des traites négrières et de l'esclavage
}

Jusqu'à la moitié $d u x^{e}$ siècle, en Europe comme en Afrique, les populations avaient tendance à adhérer aux récits officiels consolidant l'Histoire nationale des pays. Le memory boom du XXe siècle donne naissance à un nouveau régime mémoriel dans lequel les minorités revendiquent l'inscription de mémoires particulières dans les récits nationaux, influencées par les mémoires de l'Holocauste'.

Les monuments commémoratifs n'échappent pas aux contestations relatives à la manière de rappeler et d'écrire le passé. Soumis à divers enjeux (politique, social, touristique...), ces «lieux de mémoire »² peuvent devenir des " agitateurs de mémoire " ${ }^{3}$ dès lors qu'ils divisent l'opinion publique au lieu de rassembler les mémoires. L'universitaire français Bertrand WESTPHAL appelle "transgressivité " les tensions liées à l'existence d'une pluralité de représentations sur l'espace et le temps. Selon lui, «l'espace est sujet aux tensions contradictoires qui naissent de systèmes de représentations incompatibles "4. Les mémoriaux peuvent alors être envisagés comme des «tiers espaces », c'est-àdire comme une zone de contact dans laquelle se jouent les rapports de force entre plusieurs groupes sociaux ayant des discours différents.

Cette aire concentre la plus grande variété de discours. Elle permet à la parole minoritaire de s'exprimer au même titre que le discours dominant, qui y perd ses privilèges. [...] Cet espace est tout sauf homogène ; il permet la synthèse de toutes les différences, la réduction de

\footnotetext{
1 Rousso Henry, "Vers une mondialisation de la mémoire », Vingtième Siècle. Revue d'histoire, 2007 , vol. 94, n², pp. 3-10.

2 Nora Pierre (dir.), Les lieux de mémoire, Paris, Gallimard, I, La République, 1984, 674 p. ; II, La Nation, 3 vol., 1986, 665 p. ; III, Les France, 3 vol., 1993, 1034 p.

3 BRICE Catherine, "Monuments : pacificateurs ou agitateurs de mémoire » in BLANCHARD Pascal et VEYRATMASSON Isabelle, Les guerres de mémoire - la France et son histoire, Paris, La Découverte, 2008, pp. 199-208. 4 Westphal Bertrand, La géocritique : réel, fiction, espace, Paris, Éd. de Minuit, coll. «Paradoxe », 2007, p.70.
} 
certaines fractures. [...] Le tiers espace est la formulation spatiale de la transgressivité, qui est elle-même un passage (une transition, etc.) et un défi à la norme établie ${ }^{5}$.

Cette définition illustre donc les dynamiques auxquelles sont soumis les mémoriaux en dévoilant les processus de transformation et d'appropriation des monuments. Cette "transgressivité " peut s'exprimer matériellement dans l'espace (superposition d'œuvres bâties, dégradation matérielle,...) ou immatériellement (apparition de nouveaux discours sur les monuments, mise en place de manifestations temporelles dans les sites,...).

Les mémoriaux consacrés aux souvenirs des victimes de l'esclavage négrier et de la traite atlantique sont d'autant plus soumis à ce phénomène de "négociation " ${ }^{6}$ qu'ils sont apparus après une longue période d'oubli liée à la persistance de mémoires traumatiques ${ }^{7}$. Ainsi, d'une part, il faut donc attendre les années 1950 pour que commencent à apparaître, sporadiquement, des commémorations officielles de l'esclavage, d'abord aux Antilles puis en Afrique et Europe. Sur le territoire français, par exemple, les premières manifestations émergent lors de la célébration du centenaire de l'abolition de l'esclavage, en 1948. D'autre part, ces mémoires de la traite sont plurielles (car portées par des acteurs institutionnels, des chercheurs, des associations d'afro-descendants, etc.), contestées (par exemple sur le nombre de personnes déportées) et prises dans des débats publics complexes ${ }^{8}$. Sur le continent africain, la controverse apparue dans les années $2000^{\circ}$ à propos de la "Maison des esclaves " de Gorée est un exemple significatif des difficultés à faire corroborer vérité historique avec enjeux mémoriels.

Cet article interroge la manière dont les mémoires des esclavages et de la traite mobilisent les mémoriaux comme des espaces d'expressions et de revendications. Comment les mémoires collectives s'emparent-elles des mémoriaux pour se rendre visibles? Autrement dit: Quels sont les usages sociaux et mémoriels des monuments?

\footnotetext{
5 Op. cit., p.117

6 À propos des difficultés d'exposer l'esclavage, voir : VERGES Françoise, "Exposer l'esclavage ", Africultures, 2013, n 91, pp. 8-19 et Bernier Céleste-Marie, Newman Judie (dir.), Public Art, Memorials and Atlantic Slavery, London, Routledge, 2009, pp. 170.

7 VERGES Françoise, La mémoire enchaînée : questions sur l'esclavage, Paris, Michel, 2006, 204 p.

$8 \mathrm{MICHEL}$ Johann, Devenir descendant d'esclave : enquête sur les régimes mémoriels, Rennes, Presses Universitaires, coll. "Res Publica », 2015, 288 p.

9 Roux Emmanuel, "Le mythe de la Maison des esclaves qui résiste à la réalité ", Le Monde, 27 décembre 1996.
} 
Dans cet article, nous nous attacherons à percevoir les complexités et les contestations d'un de ces lieux à travers une analyse de la "Route de l'esclave " de Ouidah, choisie comme terrain d'enquête. Cette étude s'implante donc au Bénin, territoire ayant participé aux traites négrières (atlantiques et sub-sahariennes) jusqu'à la fin du XIXe siècle. Après une période de colonisation française (1899-1960), la gestion des mémoires des esclavages connaît un basculement dans les années 1980 puisqu'une mémoire nationale est progressivement édifiée dans un contexte social et politique complexe ${ }^{10}$. Plusieurs médiums sont développés pour asseoir cette "mémoire extérieure " dont l'édification d'un patrimoine bâti. L'instauration d'un circuit mémoriel, appelé "Route de l'esclave » (1993), à Ouidah, a été l'un des principaux espaces de commémoration dédiés à l'histoire de l'esclavage conçus par l'État béninois. Ce lieu a une certaine résonance à l'échelle du continent africain par son ampleur géographique $(3 \mathrm{~km})$ et par son édification ex nihilo'2. Mais depuis l'inauguration de ce lieu de mémoire, plusieurs monuments se sont superposés à ce site déjà complexe et des mémoires plurielles et familiales se sont progressivement entremêlées au discours officiel. Ces initiatives, menées individuellement ou collectivement, saturent désormais le paysage mémoriel en tentant d'y inscrire leur propre lecture du passé.

C'est donc à travers une étude sémiologique et ethnographique de la « Route de l'esclave » de Ouidah, engagée en 2015, que cet article révèle synthétiquement comment ce site devient le terrain de jeu de contestations mémorielles. En mobilisant des outils issus de l'architecture et de la géographie sociale (relevé des transformations spatiales,

10 Araujo Ana Lucia, «Mémoires de l'esclavage et de la traite des esclaves dans l'Atlantique Sud : enjeux de la patrimonialisation au Brésil et au Bénin », thèse de doctorat, Québec, Université Laval, 2007, 381 p. 11 HalbWAChS Maurice et NAMER Gérard, La mémoire collective : éd. critique par Gérard Namer, Paris, Michel, 1997, 295 p.

12 Pour faire une rapide chronologie, on note la mise en place de premiers lieux de mémoire institutionnels rappelant la traite négrière, en Afrique, dans les années 1970. Globalement, on assiste à une transformation d'anciens sites historiques en espaces pédagogiques et culturels. Dans l'ordre chronologique, on a : l'ouverture au public de la Maison des esclaves de Gorée au Sénégal (1962), la transformation de l'ancien fort portugais en Musée historique de Ouidah (1967), l'ouverture du Fort de Cape Coast au Ghana (1970), le Musée Villèle qui s'installe dans une ancienne plantation réunionnaise (1976), l'ouverture du Musée National de l'esclavage de Luanda, en Angola, dans l'ancienne chapelle (1977),... Dans les années 1990, une deuxième vague de remémoration de l'esclavage est observée, notamment impulsée par le $500^{e}$ anniversaire de la découverte du Nouveau Monde (1992) et par le programme scientifique de la "Route de l'esclave " (1994) de I'Unesco. Les lieux de mémoire alors inaugurés ne se limitent plus à des réhabilitations de sites historiques et leur matérialisation devient davantage symbolique. Ainsi, l'édification de la «Route des esclaves » de Ouidah (1993), l'inauguration d'une stèle devant l'embarcadère de Loango en Angola (1994), la mise en place de la Slave Road de Badagry au Nigeria (1999), etc. ont renforcé la matérialisation des mémoires de l'esclavage atlantique sur le continent africain. 
cartographie des nouveaux usages), et une méthodologie socio-ethnographique (observations participantes, entretiens,...), nous donnons à voir les différentes strates de constructions plastiques et narratives de ce lieu de mémoire.

\section{Matérialisation d'une mémoire à travers l'instauration d'un circuit mémoriel à Ouidah}

La «Route de l'esclave » de Ouidah, qui désigne le chemin qu'empruntaient les captifs vers les navires négriers, est patrimonialisée au début des années 1990, lorsque les autorités béninoises la transforment en un "lieu de mémoire » de la traite atlantique.

Plusieurs facteurs expliquent le choix de cette ville de Ouidah: d'abord, il s'agit d'un des ports ayant eu une activité soutenue durant la traite atlantique. Cet ancien comptoir de vente fut d'abord dirigé par la population autochtone xwéda, elle-même inféodée au royaume d'Allada' ${ }^{13}$. Suite à la conquête de Ouidah par le royaume d'Abomey (1727), c'est le peuple fon qui pilota les tractations avec les forts portugais, français, anglais et néerlandais. Ainsi, d'après Robin LAW, plus d'un million d'esclaves quittèrent le comptoir de Ouidah en direction des Amériques ${ }^{14}$. De ce fait, de nombreux écrits relatent l'histoire de ce port ${ }^{15}$, contrairement aux autres comptoirs de traites du littoral béninois moins documentés, comme ceux d'Offra et Jacquin ou Porto-Novo.

Ensuite, en Afrique, l'institution des mémoires nationales de la traite négrière s'est rapidement traduite par la valorisation d'anciens sites historiques telle que la Maison des esclaves de Gorée (1962). Le Bénin, ayant peu de vestiges bâtis témoins d'un passé négrier, se tourne vers la ville de Ouidah, encore dotée de traces architecturales ${ }^{16}$ (notamment avec la présence de l'ancien fort portugais et des bâti-

\footnotetext{
13 Jusqu'à la fin du xx siècle, le territoire qui délimite aujourd'hui les frontières du Bénin était fractionné en plusieurs royaumes, entretenant des relations de type féodal plus ou moins importantes. Les limites de ces royaumes évoluèrent constamment au gré des guerres et des politiques d'expansion.

14 LaW Robin, Ouidah: The Social History of a West African Slaving "Port ", 1727-1892, Athens, Ohio State University Press, Oxford, James Currey, 2004, 307 p.

15 Entre autres: ALLEN W, Description de Whydah et de Badagry, Paris, Annales maritimes Coloniales, 1846, pp. 18-45; Bouche Pierre (Abbé), La Côte des esclaves et le Dahomey, Paris, Plon, 1885, 426 p. et PrUnEAU De Pommegorges Antoine, Description de la Nigritie, Paris, Maradan éditeur, 1789, 284 p.; SnelgraVe William, Nouvelle relation de quelques endroits de Guinée et du commerce d'Esclaves qu'on y fait, Amsterdam, Coulanges, 1735, 316 p. Puis, plus récemment: AGBO Casimir, Histoire de Ouidah du XVI’̀ au XIX è siècle, Avignon, Les Presses Universelles, 1959, 307 p.
} 
ments construits par les Agudas ${ }^{17}$ ). La valorisation de ce patrimoine matériel fit d'ailleurs l'objet d'une première proposition du gouvernement de Mathieu KÉRÉKOU (1972-1990) ${ }^{18}$, en soumettant un projet de restauration du fort portugais de Ouidah. En parallèle, le Ministère de l'alphabétisation et de la Culture formule, en 1984, la volonté de rappeler les derniers instants des captifs sur le continent africain avant d'être vendus, ce qui évoque un terrain favorable à l'apparition des mémoires des esclavages dans l'espace public béninois ${ }^{19}$. Cette dynamique gouvernementale s'accompagne d'un mouvement local de réactivation de la ville de Ouidah. En effet, dans un même temps se constitue un collectif de notables et d'intellectuels, appelé UGDO (Union Générale pour le Développement de Ouidah) militant pour une renaissance économique et politique de la ville ${ }^{20}$.

C'est finalement sous le mandat de Nicéphore SOGLO (1991-1996) qu'une réelle impulsion est donnée par l'État en termes de politiques publiques mémorielles. En 1993, le président profite de la célébration du 500ème anniversaire de la découverte de l'Amérique par Christophe Colomb (1492) pour mettre en place le festival "Ouidah 92 - Retrouvailles Amériques - Afrique ». L'organisation de cet événement, soutenue par diverses institutions telles que I'UNESCO ou la Mission française (MCAC), est confiée à l'écrivain et poète TIDJANISERPOS, à l'époque délégué permanent adjoint à l'UNESCO. Cette manifestation, d'une dizaine de jours, met le vodoun à l'honneur et lie les deux continents à travers une pratique religieuse commune. Parmi les quatre lieux accueillant les diverses festivités (des spectacles musicaux, des foires de livres, des cérémonies religieuses...), un des sites fait explicitement référence au passé négrier du Bénin ${ }^{21}$. En

17 II s'agit de la communauté composée, entre autre, de négriers et commerçants européens puis d'esclaves revenus du Brésil après les révoltes de 1835. voy. GURAN Milton, "Du bricolage de la mémoire à la construction de l'identité sociale. Les Agoudas du Bénin », Rue Descartes, 2007, n58, pp. 67-81

18 Hass Robert, «Musée historique de Ouidah: Centre international d'études et de recherches sur la traite négrière et la diaspora noire : République populaire du Bénin», Rapport établi à l'intention du gouvernement de la République Populaire du Bénin par I'UNESCO, Paris, Unesco, 1984.

19 Dans le milieu scientifique, l'instauration d'un colloque à Cotonou par l'Unesco sur « les apports culturels des Noirs de la diaspora à l'Afrique " (1983) amorce un premier questionnement. Puis, la planification de la mise en place d'un "Centre international d'études et de recherches sur la traite négrière et la diaspora noire " par le ministère de la Culture béninois s'accompagne d'une volonté de réaliser un parcours commenté relatant le cheminement des esclaves jusqu'à la mer (1984). En parallèle, des intellectuels béninois commencent à s'approprier l'Histoire de l'esclavage sur le continent africain, jusqu'alors majoritairement rédigée par des spécialistes européens.

20 UGDO, Les voies de la Renaissance de Ouidah, Caen, Kanta, 1985, 183 p.

21 Bien que les villes de Porto-Novo et Cotonou réceptionnent quelques manifestations, l'essentiel des activités se déroule à Ouidah, dans la Forêt sacrée (Kpassèzoumè), dans la maison du Brésil, dans la maison du daagbo hounon (litt. Chef suprême de la religion vodoun) et sur la "Route de l'esclave ". 
effet, sur la route allant du centre-ville de Ouidah à l'océan, des sculptures pérennes sont inaugurées pour rappeler le départ des esclaves jusqu'aux navires négriers. Sur plus de trois kilomètres, six étapes (dont la véracité historique est remise en cause par Robin LAW ${ }^{22}$ ) structurent le parcours : (1) La "Place des enchères " qui symbolise le moment de vente des esclaves, (2) «L'arbre de l'oubli » qui marque la volonté de faire oublier aux captifs leurs racines à travers un rituel, (3) la "Case Zomaï» où les esclaves étaient stockés en attendant l'arrivée du navire, (4) le "Mémorial Zoungbondji » qui matérialise la fosse commune où étaient jetés les captifs décédés, (5) «l'Arbre du retour » qui révèle le rituel permettant aux esclaves de revenir sur leur terre natale une fois décédés, et enfin, (6) l'arrivée sur la plage qui évoque la montée dans les cales de navires et le départ pour les Amériques. Pour marquer les différentes étapes de ce circuit semi-fictionnel, le comité fait exclusivement appel à des artistes béninois à la renommée grandissante provenant du sud du Bénin ${ }^{23}$. Ainsi, l'essentiel des œuvres qui jalonnent le sentier est produit par Cyprien TokoudAGBA, hormis les statues de "Mamie Wata » et de la " case Zomaï » (de l'artiste Kouas), et la Fosse commune (réalisée par l'architecte Fortuné BANDEIRA, et les frères DAKPOGAN) ${ }^{24}$. Leur spécificité artistique ${ }^{25}$ et les références convoquées ${ }^{26}$ donnent lieu à des œuvres relativement hétéroclites tant dans la forme que dans la symbolique. Pourtant, bien que ces sculptures évoquent majoritairement les emblèmes des souverains d'Abomey ou des divinités vodouns ${ }^{27}$, c'est la mémoire des esclaves déportés qui est commémorée en rappelant les différentes étapes qui précédaient leur départ. En inaugurant ce chemin lors d'une célébration artistique et religieuse, la mémoire nationale assimile moins l'esclavage transatlantique à la notion de crime ou de déracinement qu'aux influences culturelles communes, dont le vodoun. De manière générale, l'ethnologue Gaetano CIARCIA observe, à la suite du festival,

22 LAW Robin, "Commémoration de la Traite Atlantique à Ouidah », Gradhiva. Revue d'anthropologie et d'histoire des arts, 2008, n॰ 8, pp. 10-27.

23 TALL Emmanuelle Kadya, BADET Jean-Pierre, "Artistes au pays ou ailleurs : quelques parcours remarquables. Goli, retour au pays de la confusion », Politique africaine, 1995, n 59, pp. 45-58.

24 BAY Edna G., "Cyprien Tokudagba of Abomey », African Arts, 1975, vol. 8, n 4, pp 24-29.

25 Certains travaillent le ciment en créant des sculptures ou des bas-reliefs (Yves KPEDE, Cyprien TOKOUDAGBA) alors que d'autres réalisent des œuvres plus abstraites en utilisant des mosaïques (Fortuné BANDEIRA), du cuivre (Frères DAKPOGAN) ou de l'acier (Dominique KouAs).

26 Cyprien Tokoudagba, originaire d'Abomey est habité par l'imaginaire fon alors que Dominique KouAS, artiste autodidacte originaire de Porto-Novo est imprégné par le travail de l'ethnographe Pierre VERGER à qui il sert de guide dans les années 1970. 
une forte « mise en relation entre les discours relatifs à l'histoire de la traite et les nouvelles formes de ritualisation de la religion perçue comme traditionnelle $\Perp^{28}$.

En parallèle, le gouvernement de Nicéphore SOGLO apporte une dimension plus scientifique à ce "devoir de mémoire " puisque son Premier ministre, Paulin HounTONDJ, se propose d'accueillir la conférence de lancement du projet de l'UNESCO «La Route de l'esclave ${ }^{29}$. Ce projet de l'UNESCO poursuit divers enjeux (éthiques, éducatifs et culturels) et s'attache à la vulgarisation et à la diffusion de l'histoire de la traite et de l'esclavage par un patrimoine scientifique et culturel. La transformation de la ville en lieu de mémoire est parachevée par l'inauguration de la Porte du Non-Retour, en 1995, financée par ce programme inter-gouvernemental. L'édifice est réalisé par l'architecte béninois Fortuné BANDEIRA et prend la forme d'un arc de triomphe dont l'entablement rappelle le départ des captifs. Implantée sur la plage, la Porte du Non-Retour matérialise la sixième et dernière étape du chemin qu'empruntaient les esclaves. Ce monument devient le point culminant du circuit mémoriel inauguré lors du festival "Ouidah 92 ", communément dénommé "Route de l'esclave » par assimilation aux projets scientifiques de l'UNESCO.

La tenue de ces deux manifestations consécutives, "Ouidah 92 " puis le lancement du projet onusien, introduit les mémoires de la traite atlantique et de l'esclavage dans l'arène politique béninoise. II est vrai que jusqu'à l'Indépendance du pays (1960), le passé négrier du Bénin était peu publicisé, à l'exception des milieux scientifiques, universitaires et littéraires. On peut cependant difficilement parler de période d'oubli puisque les souvenirs de l'esclavage étaient véhiculés dans les cercles familiaux, par exemple à travers des discours panégyriques lors d'obsèques. Cependant, le sociologue béninois Émile Ologoudou, issu d'une famille d'esclaves affranchis, évoque les difficultés à énoncer clairement une ancienne condition servile dans les narrations familiales, jouant alors de stratégies et de sous-entendus ${ }^{30}$. Sur le plan collectif, ces mémoires étaient moins perceptibles dans

\footnotetext{
28 CIARCIA Gaetano, « Restaurer le futur. Sur la Route de l'Esclave à Ouidah (Bénin) », Cahiers d'études africaines, 2008 , vol. 192, $n^{\circ} 4$, pp. 687-706.

29 La conférence de lancement du projet se tient le $1^{\text {er }}$ septembre 1994, à Ouidah, sur le thème : "De la traite négrière au développement : réflexion sur les conditions d'une paix durable". Elle est inaugurée par le Président du Benin, Nicéphore SogLo, et le directeur général de l'Unesco, Federico MAYOR. Le rapport final de la première réunion du comité scientifique nous indique qu'il a été décidé de donner la priorité à la route atlantique de la traite négrière. voy. DIENE Doudou, La Chaîne et le lien: une vision de la traite négrière, Paris, Éd. Unesco, 1998, 591 p. 
l'espace public, mais la pratique de l'esclavage laissa néanmoins des traces dans les arts et la culture (œuvres artistiques, chants, danses, cultes, rituels) ${ }^{31}$. Au Bénin, par exemple, le culte de Mami Tchamba est destiné à " accueillir les esprits de ceux qui sont morts en captivité , loin de leur terre et de leurs divinités ॥ $^{32}$. Ainsi, l'apparition d'une mémoire nationale des traites et des esclavages provoque un basculement dans la connaissance et la reconnaissance du passé négrier. Nassirou BAKO ARIFARI démontre que ce débat lève un tabou tout en créant " une mémoire éclatée, plurielle, parcellaire et sélective " ${ }^{33}$. Cette mémoire officielle, érigée par la classe «bourgeoise de souche esclavagiste ${ }^{34}$, élabore un discours préférant une approche cultuelle et occulte la question des responsabilités. L'institution d'un discours commun à la nation exacerbe ainsi les décalages dans la manière de concevoir cet événement.

\section{Réécriture du site et détournement de sens.}

Pendant le mandat de KÉRÉKOu (1996-2006), le Bénin continue de mener des actions visant à consolider une mémoire nationale des traites et des esclavages, mais davantage sous le prisme du pardon et de la rédemption. La décentralisation des manifestations commémoratives vers Cotonou ${ }^{35}$, la capitale économique, puis le désengagement progressif de l'État sous le mandat de BONI (2006-2016), permettent l'apparition d'initiatives personnelles ou collectives autour de la "Route de l'esclave » de Ouidah. Ainsi, au début des années 2000, outre les acteurs religieux déjà impliqués, de nouvelles catégories de population questionnent ce "devoir de mémoire " et s'emparent de ce site. Suivant des logiques disparates, leurs motivations visent à appuyer, nuancer ou contester le discours officiel. 
En 1999, le travail de réhabilitation de la ville mené conjointement par la municipalité de Ouidah (à l'époque du mandat de Pierre BADÉ) et la coopération allemande met en exergue les dissensions entre discours officiels et narrations familiales. Parmi les différents sites aménagés, la «Place des enchères » est réhabilitée. La première étape du circuit mémoriel se dote donc d'une plaque commémorative renommant le lieu "Place Chacha » ${ }^{36}$, en référence à l'ancien négrier Francisco Felix DE SouzA (1754-1849) qui dirigeait la vente d'esclaves à Ouidah au début du Xxe siècle. Cette nouvelle appellation, usitée par les Ouidahnais pour désigner cette place avant "Ouidah 92 », dénature néanmoins le message de ce premier arrêt commémoratif. Le lieu ne rend plus hommage aux esclaves, mais célèbre la notoriété du vendeur d'esclaves le plus connu du Bénin. À l'époque, l'instauration de cette plaque dans l'espace urbain avait contrarié certains descendants du négrier, qui continuent de vivre dans la demeure de leur ancêtre, derrière ladite place. "Chacha c'est un surnom, ça familiarise, ça enlève une dimension de puissance, de pouvoir à l'individu. Et c'est dans ce sens que les de Souza avaient réagi en disant: 'Mettez ou le nom, ou carrément indiquez le lieu historique' " explique Rachida DE SOUZA ${ }^{37}$. Cette anecdote révèle, d'une part, les confrontations narratives issues des différents usages historiques d'un même lieu ${ }^{38}$. D'autre part, on perçoit la difficulté à assumer certaines mémoires à Ouidah. Le film "Mémoire promise "39, qui interroge notamment Martine DE SouzA, descendante du commerçant portugais, illustre l'ambivalence entre l'attitude de glorification d'un aïeul reconnu et de culpabilité face aux condamnations morales actuelles de l'esclavage. 
Figure 1. Photomontage schématique représentant la superposition d'installations et de monuments commémoratifs le long de la voie menant à l'océan depuis le centre-ville de Ouidah.
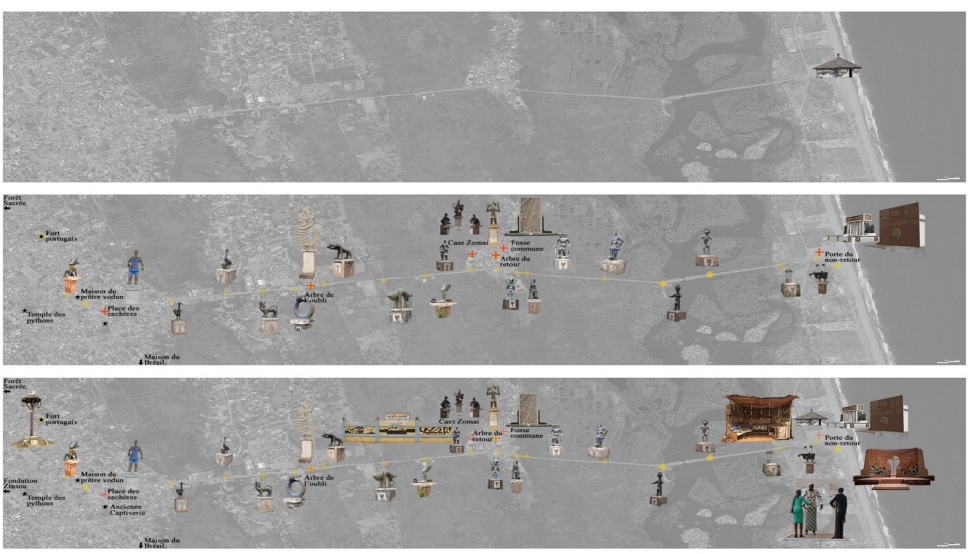

Planche I: Avant 1993, il existait déjà un autel sur la plage / Planche II (1995) : Inauguration de la "Porte du NonRetour » face à l'océan qui complète les sculptures installées durant le festival “ Ouidah 92 » (1993) / Planche III (19982012): Instauration de nouveaux édifices le long du parcours (Mémorial Zomachi (1998), Mémorial du grand jubilé de l'an 2000 (2000), Porte du retour (2004), Assin géant (2012)) et d'échoppes commerciales.

Copyright : Rossila Goussanou

L'année suivante, c'est l'extrémité sud du parcours, du côté de la plage, qui est l'objet d'une re-sémantisation. Plusieurs travaux démontrent que la présence de l'océan au Bénin (et de ce qui est arrivé par l'océan) est vecteur d'imaginaires et de croyances encore très actifs. Ainsi, certains rattachent le développement du culte de Mami Wata, esprit aquatique vénéré tout le long du golfe de Guinée, à la présence des navires européens le long des côtes africaines durant la traite atlantique ${ }^{40}$. De même, la prière séculaire durant laquelle le Daagbo hounon (le chef suprême de la religion vodoun) rejoint la mer chargé de précieux objets indique la haute sacralité de ce lieư ${ }^{4}$. C'est sur ce même littoral ouidahnais que l'Église catholique implante, face à l'océan, le «Mémorial du grand jubilé de l'an 2000 " afin de célébrer l'arrivée des premiers missionnaires sur les côtes béninoises. Les premiers évangélistes, arrivés peu de temps après l'arrêt de la traite atlantique (en 1877), sont venus christianiser la population locale. La pratique du vodoun, si elle est célébrée par SoGLO, a longtemps été récriminée, et ce en partie par les missionnaires. Le monument présente une assemblée de croyants rendant hommage à la Trinité. La

40 Bortolotto Chiara, "La sirène Mami Wata : un cas de réemploi transculturel ", L'Autre, 2009, vol. 10, $\mathrm{n}^{\circ} 1$, p. 37.

41 TALL Emmanuelle Kadya, " Guerre de succession et concurrence mémorielle à Ouidah, ancien comptoir de la traite ", Politique africaine, 2009, vol. 115, n³, pp. 155-173. 
couleur ocre, la présence d'un socle et le percement sur l'océan reprenant les frontières du pays rappellent la Porte du Non-Retour, située à quelques mètres. Cependant, alors que le monument de l'UNESCO déplore l'aspect tragique des premiers "échanges commerciaux " effectués avec l'Occident, le mémorial religieux acclame la venue des premiers missionnaires colons.

Dans les mêmes temps, deux autres édifices conséquents, à l'initiative d'intellectuels béninois, altèrent de manière significative le sens premier de ce circuit. En 1998, l'Institut de Développement et d'Echanges Endogènes (IDEE) ${ }^{42}$, coordonné par le sociologue béninois Honorat AGUESSY, acquiert une parcelle jouxtant la " case Zomaï » (troisième étape du circuit). Un complexe culturel et hôtelier appelé «Mémorial Zomachi - Cité de la Diaspora - Place du devoir de mémoire sur l'esclavage " est édifié et modifie fortement l'aspect paysager du site. Le long de la façade extérieure, une série de bas-reliefs retrace l'histoire de l'esclavage à travers le parcours des esclaves noirs, depuis leur capture sur le continent africain jusqu'à leur émancipation. "La Porte-du-Non-retour n'est pas une initiative du Bénin, c'est une initiative de l'UNESCO " explique Honorat AGUESSY, démontrant que cette réponse architecturale n'est pas suffisante pour répondre aux enjeux locaux ${ }^{43}$. Lui préfère rendre hommage aux "héros noirs " contemporains tels que Malcolm X ou Kwame KHRUMA et inviter la population locale à "demander pardon » à cette diaspora issue de la traite atlantique ${ }^{44}$.

En 2004, un nouvel édifice appuie la logique de repentance amorcée par AGUESSY. L'organisation Pro.Me.Tra, présidée par le médecin Eric GBODOSSOU, inaugure un espace muséographique visant à accueillir, une fois par an, des descendants d'esclaves. Il fait également, à travers différentes œuvres, une chronologie de la présence des Noirs en Amérique, de l'esclavage à la ségrégation. Ce lieu, intitulé "La Porte du retour ", semble s'opposer à la "Porte du Non-Retour ", située à quelques centaines de mètres. II contient une stèle commémorative dévoilant une femme portant une tenue en wax (représentant la mère Afrique) qui accueille à bras ouverts ses enfants (un homme et une femme) habillés de manière occidentale. Cette compo-

42 L'IDEE est implanté à Ouidah dans les années 1990. II a pour objectif de promouvoir les savoirs locaux à travers la mise en place d'activités scientifiques, culturelles et pédagogiques. voy. http://www.idee-benin.com 43 Extrait d'entretien réalisé le 23/01/2016 avec Honorat AGUESSY

44 Une première « marche du repentir » est organisée en avril 1998 par l'IDEE. Cet événement, aujourd'hui annualisé, consiste à assumer collectivement les « responsabilités morales » de l'esclavage en demandant pardon aux membres de la diaspora africaine. 
sition artistique opère un saut historique entre le circuit mémoriel qui fait référence aux siècles de traite, et le retour des afro-descendants sur le continent africain.

Gaetano CIARCIA interprète le travail de ces "entrepreneurs de mémoire " comme une manière d'interférer avec "ce que l'itinéraire officiel de la Route de l'esclave risque d'occulter : la possibilité de rendre ostensible hic et nunc un retour physique et 'spirituel' (et pas seulement symbolique) » ${ }^{45}$. De manière plus générale, l'apparition de ces nouveaux schèmes interprétatifs s'opère pendant que la mémoire officielle bascule. Durant le mandat de KÉRÉKOu, les activités commémoratives associent ce "retour physique " de la diaspora vers le Bénin à des enjeux de développement économique, comme l'atteste la création d'une structure gouvernementale, en 2001, appelée "Association Béninoise pour la Réconciliation et Développement ". La présence du Président KeREKOu lors de la première édition de la "marche du repentir ", organisée par Honorat AGUESSY en 1998, appuie cette reconversion et illustre le fait que ces initiatives pouvaient être en adéquation avec les politiques publiques mémorielles de l'époque.

On note également, en marge du parcours, une nouvelle intervention de la municipalité ouidahnaise en 2012. Aux abords du musée d'Histoire de la ville, qui prit place dans l'ancien fort portugais en 1967, un nouveau lieu de mémoire est inauguré pour «symboliser l'immortalité des millions de Noirs d'Afrique déportés dans le honteux commerce triangulaire et dont le sacrifice des âmes exige de nous, repenti et compassion de tous les instants » et pour "rappeler aux civilisations occidentales leur devoir de mémoire et leur dette vis-à-vis de nos ancêtres de Ouidah, du Bénin et de l'Afrique ${ }^{46}$. La plaque commémorative est accompagnée d'un assin. Cette forme de représentation des morts, une tige de fer surmontée d'un disque métallique, est typique des autels funéraires fons. Ce nouvel élément, implanté à quelques mètres de la "Place des enchères " s'oppose à la figure d'egun (également appelée « revenant »), un emblème récurrent dans la patrimonialisation de la Route de l'esclave ${ }^{47}$. Ce symbole, qui évoque le retour des âmes dans les familles Yorubas (groupe social qui est localement associé, par généralisation, aux esclaves les plus vendus à Ouidah), est apposé de part et d'autre de la Porte du Non-Retour ${ }^{48}$. 


\section{Entre apparition, disparition et conservation des euvres artistiques}

À cette liste (non exhaustive) d'initiatives de grande ampleur s'ajoutent des revendications plus ponctuelles. Sur une échelle de temps plus réduite, ces manifestations viennent néanmoins altérer l'intégrité du circuit mémoriel. Parmi les interventions artistiques et temporaires, le travail d'Edwige APLOGAN est particulièrement connu ${ }^{49}$. En 2012, cette artiste béninoise recouvre l'arbre de la «Place des enchères " par les drapeaux de pays africains et la rebaptise la "Place des mille douleurs ». Cette réalisation s'inscrit dans une série d'installations, principalement liées à l'histoire de l'Indépendance africaine, où elle recouvre les lieux de mémoire de drapeaux. Elle interroge ainsi l'unité africaine et donne une autre dimension à l'arbre, premier «lieu de mémoire » africain. En remettant en valeur les bâtiments, l'artiste ne propose plus une nouvelle lecture du passé, mais amène les populations locales à re-questionner leur symbolique.

Contrairement à son homologue béninoise, le plasticien AROLANDO emploie un vocabulaire très figuratif pour réaliser, en contre-bas de la Porte du Non-Retour, l'installation «Commerce triangulaire ». Cet artiste originaire d'Abomey, "conscient d'un passé riche mais douloureux ${ }^{50}$, travaille à partir de matériaux de récupération. En 2014, dans le but de représenter l'histoire et rendre plus vivant le souvenir de la traite atlantique, il met en place une série de silhouettes implantées dans le sable. Les scènes représentent les tractations effectuées entre les dirigeants africains et les négriers européens et dénoncent l'aspect lucratif de ce commerce. Une seconde installation artistique, composée de sculptures caricaturant les convois d'esclaves, interroge et met en tension la présence d'emblèmes royaux aboméens le long du parcours.

Si un certain nombre d'objets se rajoutent progressivement au circuit mémoriel initial, d'autres œuvres disparaissent, partiellement ou totalement. Malgré les différentes réhabilitations du site (en 1999, 2006 et 2010) qui modifient considérablement l'aspect chromatique des statues qui composent le parcours ${ }^{51}$, plusieurs œuvres sont détériorées. Par exemple, les statues représentant des Amazones, les 
femmes guerrières qui étaient destinées à se battre durant les conflits inter-royaux, sont régulièrement destituées des armes qui permettent de les caractériser. Pareillement, jusqu'en 2016, la statue représentant une hyène pour certains, le symbole du roi fon Glèlè (1858-1889), une panthère pour d'autres, est décapitée. Quant aux colombes qui encadraient la Porte du Non-Retour, elles disparaissent moins d'une dizaine d'années après leur implantation. Les textes descriptifs des œuvres sont également effacés à l'aide de pierre pour conforter la présence de guides aux abords du circuit mémoriel afin de donner des explications aux visiteurs. Si certaines œuvres sont détruites suite aux mauvais entretiens ou convoitées pour leur valeur pécuniaire (notam-

Figure 2. "Place des enchères » de Ouidah.

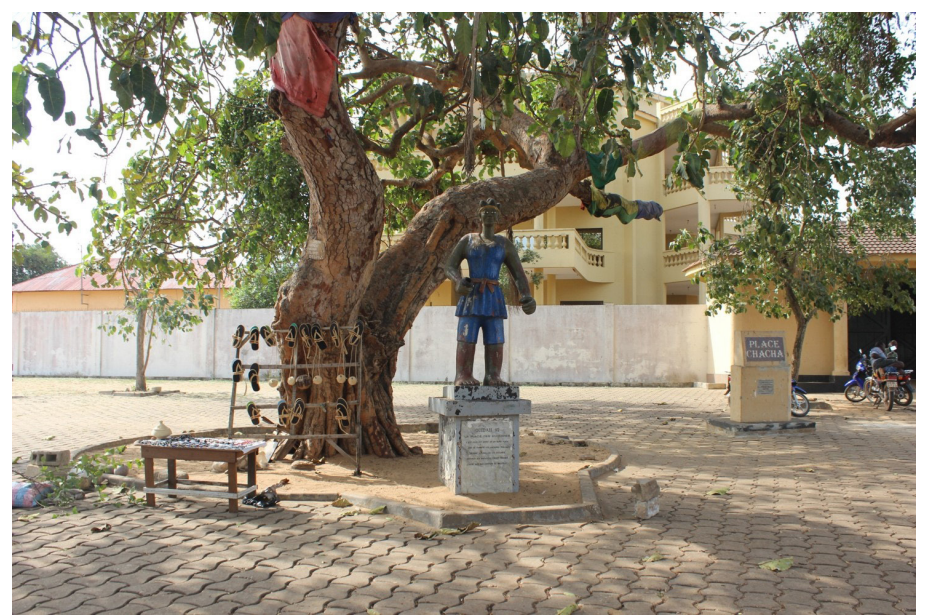

Au premier plan se trouve la statue représentant une amazone, réalisée par le plasticien Cyprien TOKOUDAGBA. Les objets qui étaient placés dans ses mains ont disparu. L'espace est investi par des supports marchands. Sur l'arbre, quelques drapeaux, installés en 2012 par Edwige APLOGAN, sont encore présents. En arrière plan, la stèle commémorative en l'honneur de Chacha est visible, surplombée par la demeure des héritiers du commerçant brésilien. Photo: Rossila Goussanou, février 2017.

ment les œuvres en cuivre et en plomb), ces dégradations peuvent également être entendues comme des actes revendicatifs. Ainsi, pour Romuald TCHIBOZO ${ }^{52}$, les œuvres d'art réalisées le long de la « Route de l'esclave " ravivent des tensions latentes entre la population originelle xwéda et la société fon, qui s'installe à Ouidah après la victoire

52 Tснівоzo Romuald, "Pratiques esthétiques sur la route de l'esclave à Ouidah : regard tourné vers le passé», in Actes de la conférence de l'ICOM "La Traite et la Route des esclaves. Sites historiques, archéologiques et mémoriaux », 24-27 novembre 2014, Marseille. 
d'Abomey en 1727. Cet historien de l'art béninois défend l'idée que la patrimonialisation de la route impose, une nouvelle fois, un imaginaire iconographique fon dans l'espace public. Cette « domination symbolique " expliquerait les actes de vandalisme sur les œuvres présentes le long de la "Route de l'esclave».

Figure 3. Transformation des colonnes de la «Porte du Non-Retour " (réalisée par Fortuné BANDEIRA et Yves KPEDE) en un espace mercantile. Photo: Rossila Goussanou, février 2017.

Figure 4. Statue détériorée, réalisée par Cyprien Tokoudagba en 1993. Photo: Rossila Goussanou, janvier 2015
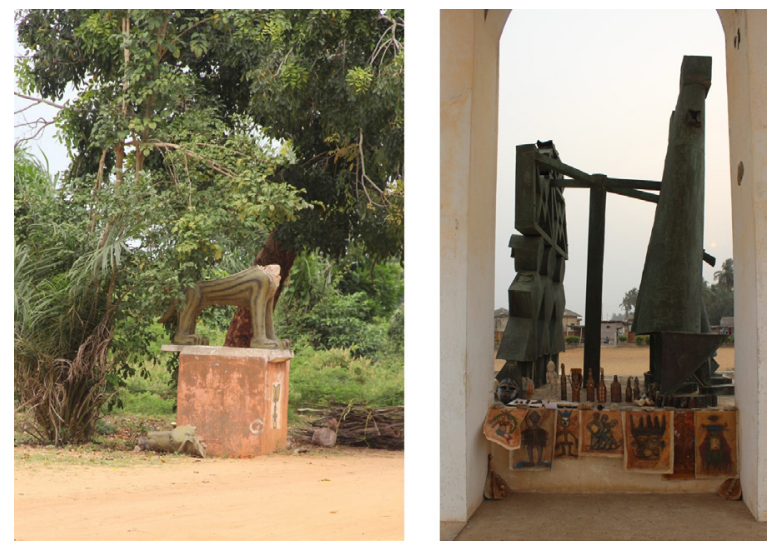

\section{Réception et incorporation des enjeux patrimoniaux par la population locale.}

Outre les interventions de type mémoriel, la "Route de l'esclave » est progressivement investie par d'autres types de constructions. Leurs motivations sont davantage de l'ordre économique et touristique que commémoratives. Effectivement, dans les années 2000 , l'amplification d'un dark tourism met en exergue la manne économique que représentent les lieux de mémoire. À Ouidah, les proches abords du circuit mémoriel sont convoités en raison de la forte affluence de touristes. Ainsi, les anciens locaux de la douane, datant de l'époque coloniale, font l'objet d'un projet touristique. Dans le but de proposer une offre hôtelière aux visiteurs, un promoteur construit des bungalows allant jusqu'aux abords de la Porte du Non-Retour. Le projet, pourtant déjà bien avancé, a finalement été interrompu par les autorités publiques. De manière générale, des structures marchandes, des étals et des échoppes vont progressivement s'implanter aux alentours 
de la plage. Le socle de la "Porte du Non-Retour " est même exploité comme présentoir pour accueillir des objets d'arts destinés à être vendus aux touristes.

L'existence de " contre-usages » de la part d'acteurs locaux traduit des manquements dans la gestion du site. L'État, propriétaire du foncier, n'exerce pas un contrôle assidu des installations, plus ou moins conformes, entreprises sur ce site, bien qu'à partir de 2007, des mesures de conservations sont prises afin de protéger le patrimoine de la ville de Ouidah, dont les œuvres de la "Route de l'esclave ". Néanmoins, ces dégradations génèrent un questionnement quant à la réception des œuvres qui jalonnent la "Route" par le reste de la population. L'hypothèse d'une " patrimonialisation à l'occidentale " qui bousculerait la manière de représenter et de se remémorer le passé peut être formulée ${ }^{53}$. Au Bénin, la gestion du passé s'effectue au travers d'un culte des ancêtres très hiérarchisé et un système de croyance très ritualisé. Le passé n'est donc pas incarné à travers l'édification de monuments, mais plutôt par la pratique de cérémonies visant à donner à un lieu, de manière temporaire, un caractère sacré ${ }^{54}$. L'architecte français Alain SINOU explique que la patrimonialisation d'anciens sites historiques n'est pas forcément bien perçue par la population locale, qui n'octroie pas de charge affective particulière à ces $\operatorname{sites}^{55}$. À Ouidah, on note que le dispositif scénographique correspond davantage à des normes occidentales (instauration de monuments et de statues, présence de socle,.... $)^{56}$. Ainsi, l'appropriation de la "Route de l'esclave " par les Béninois découlerait plus d'un rapport au paysage, que de celui d'un mémorial, puisque ce site devient un lieu de promenade ou un espace mercantile. Le fait d'usiter ce lieu dans un but lucratif, en y plaçant des étalages et des magasins de souvenirs, n'a pas un caractère offensant ou péjoratif. Cependant, les usages quotidiens de ces sites entrent en contradiction avec la dimension internationale et inaliénable que leur attribuent les autorités nationales, comme le démontre la volonté d'inscription de la "Route de l'esclave » au patrimoine mondial de l'UNESCO, demande jusqu'à présent refusée.

53 Corallı Monica et HouÉnOudé Didier, «La patrimonialisation à l'occidentale et ses conséquences sur un territoire africain », Espaces et sociétés, 2013, vol. 152-153, n¹, pp. 85-101. 


\section{Tabula rasa?}

La proposition du nouveau président de la République du Bénin, Patrice TALON, élu en avril 2016, semble neutraliser la superposition d'artefacts, pérennes ou temporaires, qu'a connue la « Route de l'esclave " de Ouidah. En effet, parmi les 7 projets phares de son programme présidentiel, la volonté de «Recréer à l'identique la cité historique de Ouidah $»^{57}$ est mentionnée. L'objectif annoncé est de faire du tourisme l'un des deux axes majeurs de création de richesses et d'emplois dans le pays.

Bien que les éléments de la future «Route des esclaves» ne soient pas encore validés, certains documents préfigurent le prochain agencement de ce lieu de mémoire ${ }^{58}$. La proposition d'aménagement est ainsi soumise à des enjeux mémoriels et touristiques. Concernant le circuit global, si les 6 étapes principales de la route seraient conservées (La place des enchères, l'arbre de l'oubli, la case Zomaï, la fosse commune, l'arbre du retour et la Porte du Non-Retour), l'idée serait de supprimer les statues pour proposer un parcours plus documenté, afin de redonner un caractère historique au site (conserver l'aspect "terreux " du chemin, reconstruire le fort français dans le centre de la ville (détruit en 1908),... ).

Figure 5. Image de synthèse présentant la réhabilitation de la «Route de l'esclave»,

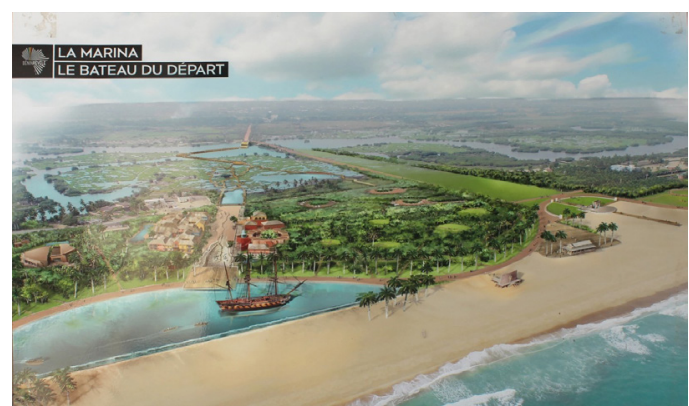

Cette projection est affichée sur la devanture de l'Agence Nationale en charge du projet (ANPT), à Cotonou. Photographie : Rossila Goussanou, mars 2018.

57 " Tourisme - 'Faire du tourisme une filière de développement économique créatrice de richesses et d'emplois' ", Site de Patrice Talon, disponible à l'adresse: https://patricetalon.com/portfolio-posts/tourisme/ (consultée le 9 mai 2018). 
De manière assez succincte, cette nouvelle scénographie proposerait aux visiteurs, non plus d'expliquer à travers des œuvres symboliques et artistiques, les derniers kilomètres que réalisaient les esclaves avant d'embarquer sur le bateau, mais de revivre cette expérience dans son contexte historique. L'historienne Maryline CRIVELLO questionne les effets de la reconstitution, cette forme " de re-création d'un fait attesté par l'Histoire ${ }^{59}$. Selon elle, ces "réincarnations du passé ", dont la véracité de la mise en scène est autant à interroger que l'Histoire elle-même, témoignent d'un nouveau clivage entre les travaux des historiens (en quête d'objectivité et authenticité) et leurs appropriations par le public (à la recherche d'une réappropriation « vécue » de l'histoire de leurs ancêtres). Ces nouvelles formes de transmission de l'Histoire, à travers un "jeu de rôle grandeur nature ", témoignent d'un nouveau rapport au passé qui s'appuierait moins sur des célébrations publiques qu'à travers une spectacularisation de l'histoire.

\section{Les mémoriaux, des espaces de négociations ?}

Ce travail d'enquête met en exergue le mécanisme complexe qui entoure le terrain béninois et renvoie à l'analyse de Maurice HALBWACHS sur l'importance d'ancrer les mémoires collectives dans un dispositif spatial afin de stabiliser et immobiliser une réalité, fusset-elle valable pour un seul groupe de personnes ${ }^{60}$. Sur les 27 œuvres initialement inaugurées en 1993 se superposent, en 25 ans, plus d'une dizaine d'autres artefacts qui perturbent le discours initial du circuit mémoriel. Ces contre-usages sont observés sur les œuvres mêmes, sur le circuit mémoriel et à ses proches abords. Ils sont menés par une multitude d'acteurs (institutions publiques, organisations locales, milieu associatif, regroupements familiaux, acteurs privés...), pris entre intérêt personnel et objectif commun et donc soumis à des enjeux divergents (mémoriel, politique, social, économique,...). Leur appropriation du site montre la violence symbolique et narrative dans laquelle se déroulent les rapports de force. La chronologie des différentes reconfigurations du circuit mémoriel de Ouidah traduit donc le caractère instable du mémorial. En effet, l'analyse historique et plastique de la « Route des esclaves " démontre à quel point ce monument fluctue, à la fois dans les discours que l'on porte sur ce lieu et ces mémoires, mais également au niveau des éléments physiques qui le composent. La création, la

59 CRIVello Maryline, "Comment on revit l'Histoire ", La pensée de midi, 2000, n³, pp. 69-74. 
suppression, la reproduction, l'apparition, la dégradation des artefacts qui constituent la "Route des esclaves " révèlent l'influence du temps sur l'espace. L'architecte-urbaniste Bernard HUET explique que cette variabilité caractérise les espaces bâtis : "l'architecture est discontinue dans le temps et dans l'espace. [...] Elle est fragmentaire, limitée et toujours inachevée car elle ne peut jamais prétendre à la permanence; elle est soumise au jeu permanent des modifications et des substitutions $"^{6}$. Les lieux de mémoire institutionnalisés sont particulièrement soumis à ce type de contestation, du fait qu'ils sont supports d'une mémoire officielle. Identifier les mémoriaux comme tels, c'est défendre l'idée qu'ils soient des "espaces publics ", au sens habermassien du terme ${ }^{62}$, c'est-à-dire des sphères dans lesquelles les opinions privées peuvent être rendues publiques. Ces espaces deviennent des lieux de confrontation entre mémoire collective et mémoire individuelle, entre discours national et discours divergent.

Plus généralement, l'analyse temporelle de «La Route de l'esclave » permet de comprendre comment la mémoire de la traite atlantique se renouvelle aujourd'hui au Bénin. La nouvelle proposition concernant la "Route de l'esclave » annonce un tournant drastique dans l'aménagement de ce site qui s'est constitué, au fil du temps, de divers monuments. Pas loin de faire tabula rasa de toutes les initiatives précédentes, le nouveau projet de TALON interroge les occultations que présage la prochaine configuration du parcours mémoriel, et permet d'analyser comment cette mémoire est à nouveau mise à l'honneur, 25 ans après la première édification institutionnelle. L'analyse des objets qui sont sauvegardés sur le site, et ceux que l'on décide d'évacuer, sont autant de témoins de la manière dont se construit une mémoire. Que faire de ces «fictions mémorielles » qui prennent de plus en plus d'ampleur dans l'espace public béninois? 